\title{
AN ENERGY EFFICIENT HIERARCHICAL ROUTING SCHEME FOR WIRELESS SENSOR NETWORKS
}

\author{
Alaa Aadri $^{1}$ and Najlae Idrissi ${ }^{2}$ \\ University of Sultan Moulay Slimane, Faculty of Sciences and Techniques \\ Information Processing Decision Support Laboratory \\ P.B. 523, Beni Mellal, Morocco
}

\begin{abstract}
Wireless sensor networks represent a very interesting field in the Internet of objects (IoT) and in information and communication technologies in view of the diversity of their applications based on the manipulation of information through sensor nodes with limited characteristics (low storage capacity, autonomous source of energy, limited power, etc.). In this kind of networks generally called WSN, hierarchical routing has shown over the last few years his increasing interest among researchers in the field of computer research aiming to guarantee energy efficiency of the network. In this paper, we compare some existing energy efficient clustering routing protocol with a new clustering protocol which we have developed during our researches using different simulation parameters, and we show that our routing protocol gives better results and extends the network lifetime.
\end{abstract}

\section{KEYWORDS}

IoT; WSN; hierarchical routing; energy efficiency; performance analyzis

\section{INTRODUCTION}

The latest developments in the fields of information technology and telecommunications have led to a massive deployment of ad hoc wireless networks that have become a key of the Internet Of Things (IOT). In WSN, nodes can be deployed to capture, store, process, and transfer the sensed data permanently or also between physical contexts and virtual universes to help in operational decision-making. The reduction of energy consumption and the self-organization of the nodes in the network are the two most studied themes in the scientific literature aiming to minimize energy consumed in the operations of capturing, processing and sending information via radio waves and to guarantee the overall functioning of the network. Clustering is among the most efficient routing techniques to overcome the constraints imposed WSN and to achieve energy balancing in the network. The rest of this paper is organized as follows: Section 2 gives brief state of the art of WSN. Section 3 describes routing in WSN and a classification of the different routing protocols classes, Section 4 presents our hierarchical routing protocol. And finally, Section 5 shows simulation parameters and performance metrics we have used and presents results and discussions. 


\section{STATE OF THE ART OF WIRELESS SENSOR NETWORKS (WSN)}

\subsection{Presentation of WSN}

A wireless sensor network consists of a set of electronic devices (sensors) capable of measuring physical values, processing them and transmitting them to a control center via a base station. Each sensor contains essentially four main units:

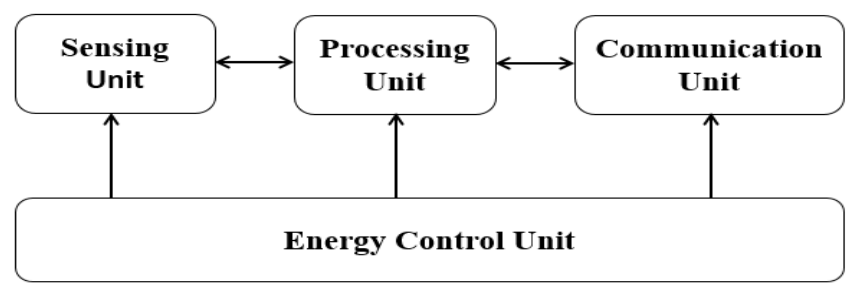

Figure 1. Sensor node components

- Sensing unit: usually consisting of a physical capture device that capture measurements and convert them into analog signals and of Analog-Digital Converters (ADCs) that convert these analog signals into digital signals.

- Processing unit: controls the procedures that enable the node to collaborate with other nodes to perform the acquisition tasks and store the collected data.

- Communication module: composed of a transceiver enabling communication between the different nodes of the network via a radio communication medium.

- Battery: the single source of energy that is generally neither rechargeable nor replaceable, it represents the main constraint while designing routing protocols for sensor networks.

\subsection{Modelization of WSN}

WSN can be modeled by a graph:

$$
G_{t=}\left(V_{t}, E_{t}\right)
$$

Where V represents the set of sensor nodes and E models all connections between these nodes. According to the organization of sensors in the deployment field, WSN can be presented under two main topologies:

- Flat topology: all the nodes are homogeneous and identical in terms of capacity and characteristics except the sink, which is responsible for the transfer of data collected to the end user. This topology allows high fault tolerance but it suffers from low scalability.

- Hierarchical topology: In this topology, nodes are divided into several levels of organization and responsibility. Clustering represents one of the most used methods; it aims to divide the network into clusters composed of a Cluster Head $(\mathrm{CH})$ and its cluster members that transfer their collected data for aggregation and transmission to the base station (BTS). This topology increases the scalability of the system, but it causes Cluster Heads overload and an unbalance in the energy consumption on the network. 


\subsection{Energy consumption model for communication in WSN}

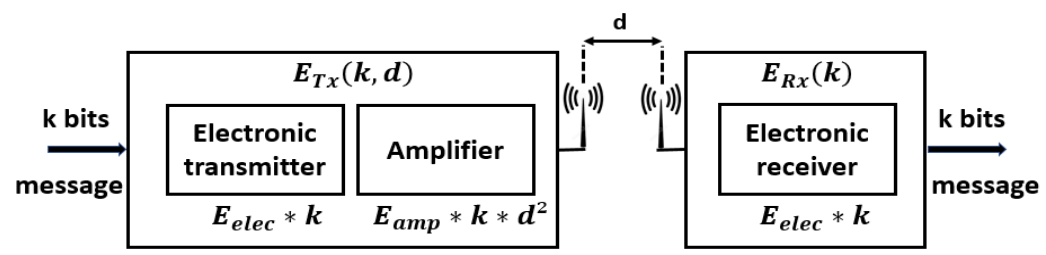

Figure 2. WSN Energy Consumption Module

In the literature, an energy consumption model was proposed in [Heinzelman \& al, 2000] (Figure 2.) to describe the energy consumed by the sensors in each operation: the emission energy consumed to capture data and the communication energy that groups the transmission energy and the reception energy:

- Transmitting energy: to transmit a message of $\mathrm{k}$ bits to a receiver far from $\mathrm{d}$ meters, the transmitter consumes:

$$
\begin{aligned}
& E_{T x}(k, d)=E_{T x \text { elec }}(k)+E_{T x \text { amp }}(k, d) \\
& E_{T x}(k, d)=\left(E_{\text {elec }} * k\right)+\left(E_{\text {amp }} * k * d^{2}\right)
\end{aligned}
$$

- Reception energy: to receive a message of $\mathrm{k}$ bits the receiver consumes:

$$
\begin{aligned}
& E_{R x}(k)=E_{R x \text { elec }}(k) \\
& E_{R x}(k)=E_{\text {elec }} * k
\end{aligned}
$$

Where $E_{\text {elec }}$ represents the electronic transmission energy and $E_{a m p}$ represents the amplification energy.

Both transmission and reception energies are determined by the amount of data to be communicated, by the transmission distance and by the physical properties of the radio module.

\section{ROUTING IN WSN}

Data routing designs the way how information is routed to its destination through a network connection, it consists on optimal packets delivering through the network using the least possible resources and ensuring network fault tolerance. In this part, we present a classification of WSN routing protocols with a focus on those based on the network hierarchization and on which a performance study will be applied in the next part of this article.

\subsection{Classification of WSN routing protocols}

Data Routing have attracted a lot of interest among the researchers, many routing protocols have been presented depending on type of application and on data routing strategies. 


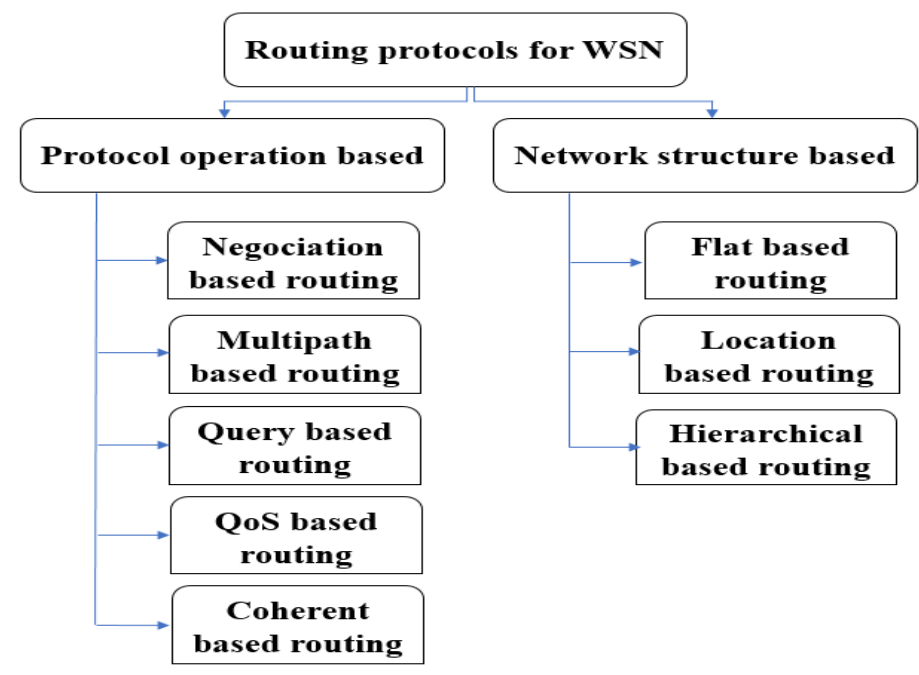

Figure 3. WSN Routing protocol classification

As shown in Figure 3. WSN routing protocols in WSN can be divided to:

- Negotiation-based routing: routes selection is based on the available resources to eliminate the redundancy of data in the network.

- Multipath-based routing: multi paths are used rather than single paths, which increases the fault tolerance but also energy consumption and traffic generation.

- Query-based routing: the destination node propagates a request for particular information in the network and the nodes possessing it respond by sending it to the requesting node.

- QOS-based routing: QoS-aware protocols consider end-to-end delay requirements while setting up the routes in the sensor network.

- Coherent-based routing: forwards data after processing and redundancies elimination to the aggregator nodes in order to improve energy efficiency

- Flat-based routing: In this routing scheme nodes are identical (in terms of battery and hardware complexity), the disadvantage is that scalability becomes critical for a very large number of sensor nodes, hence the need to manage and organize the nodes using access control medium (MAC).

- Location-based routing: uses location information to guide route discovery and data transmission. It allows an optimized routing at reduced cost but the disadvantage is that each node must know the location of the other nodes of the network.

\subsection{Hierarchical routing in WSN}

Hierarchical-based routing represents one of the most efficient strategy to improve energy efficiency and to achieve self-organization of the network. Several routing strategies have been proposed in the literature, in this paper we focus on the following energy efficient hierarchical routing protocols:

\subsubsection{LEACH (Low-Energy Adaptive Clustering Hierarchy)}

This protocol was proposed by ChandraKasan \& al. to provide an efficient solution to the problem of energy consumption in the WSN, it is based on the formation of clusters in which the elected $\mathrm{CHs}$ collect and aggregate the data captured by their cluster member nodes to 
subsequently transmit them to the base station, a $\mathrm{CH}$ performs its role as cluster leader for a period of time called "round", at the beginning of each round each node of the network determines whether it wants to be a $\mathrm{CH}$ by calculating a number between 0 and 1 if this number is less than a threshold $\mathrm{T}(\mathrm{n})$ the node becomes $\mathrm{CH}$, the threshold $\mathrm{T}(\mathrm{n})$ is expressed by the relation:

$$
T(n)=\left\{\begin{aligned}
\frac{p}{1-p\left[\operatorname{rmod}\left(\frac{1}{p}\right)\right]} & \text { if } n \in G \\
0 & \text { if not }
\end{aligned}\right.
$$

With p: the percentage of $\mathrm{CHs}$ in the network; r: the number of the current round; G: the number of nodes that have not been selected as $\mathrm{CHs}$ in the previous $1 / \mathrm{p}$ rounds. Once the clusters are formed, each $\mathrm{CH}$ sends its identifications to the nodes of the network through the CSMA protocol and assigns to each member node of its cluster an interval of time during which it can send its data based on the TDMA approach.

\subsubsection{PEGASIS (Power-Efficient Gathering in Sensor Information Systems)}

This routing protocol is considered as an optimization of LEACH, it gathers the network nodes in a long chain based on the principle that a node can communicate only with the closest node to it. Thus, each node adjusts its radio for a very short communication to conserve its energy. To communicate with the Sink, the process is organized into rounds; during each round a single node is allowed to communicate directly with the sink. This privilege is granted to all the nodes of the network in turn. A better conservation of energy is obtained by the data aggregating on each node of the network: in each round, only one node can communicate directly with the sink, it's called "leader node", this privilege is given in turn to all the nodes of the network. Nodes transmit their data throw their neighboring nodes toward the leader node that sends it afterward to the base station.

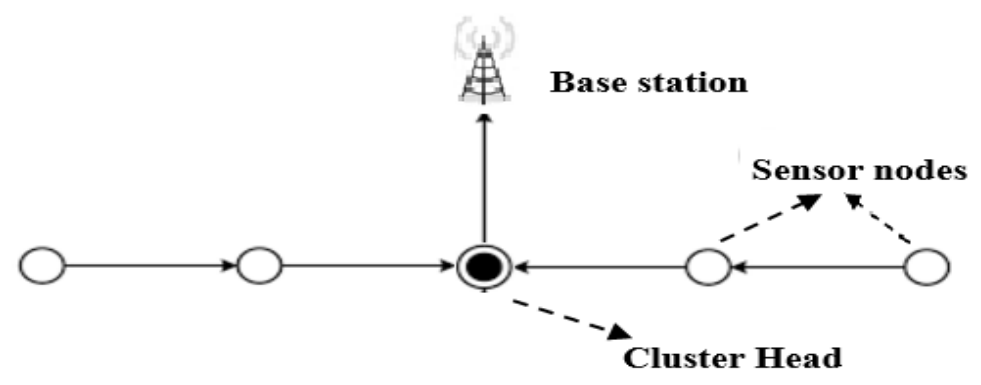

Figure 4. Chain construction in PEGASIS

\subsubsection{HEED (Hybrid Energy-Efficient Distributed clustering)}

This protocol aims to divide the network into one hop clusters jump where $\mathrm{CHs}$ are elected according to two metrics: energy and cost of paths, each node calculates its probability to become $\mathrm{CH}$ by the following formula:

$$
C H_{\text {prob }}=C_{\text {prob }} *\left(E_{\text {Res }} / E_{\text {Max }}\right)
$$

Where $E_{\text {Res }}$ represents the remaining energy of the node and $E_{\text {Max }}$ its initial energy. $C H_{\text {prob }}$ is always greater than a threshold $p_{\min }$ Inversely proportional to $E_{\operatorname{Max}}$. 
A node of the network can be presented under two states: "tentative status" if $\mathrm{CH}_{\text {prob }}<1$ Or

"final status" if $\mathrm{CH}_{\text {prob }}=1$.

\section{PROPOSED PROTOCOL FOR HIERARCHICAL ROUTING IN WSN}

\subsection{Description}

Our hierarchical routing scheme intends to form interconnected clusters through discovery messages exchange between the nodes and to use a TDMA technique to set a sleeping mechanism for sensors in order to extend the network lifetime, it integrates also a method for data aggregation based on multi-hops intra-cluster and inter-cluster communications aiming to satisfy the compromise "Energy consumption - Quality of Service (QoS)" .

\subsection{Phases of our routing scheme}

\subsubsection{Initialization phase}

Initially the nodes are in listening mode, the clusters will be defined by a diffusion technique controlled by the BTS which broadcasts a discovery message on the covered zone containing a node identifier fixed at ' 0 ' and incremented each time by the receiver node before sending it to its neighbors, and also a cluster identifier incremented only by the $\mathrm{CH}$ nodes, these identifiers are manipulated so as to construct clusters with 2 hops gradually with the $\mathrm{CH}$ node as a center.

\subsubsection{Clustering phase}

we denote by $n$ a single node in the network and by $N$ the total number of the nodes in the network, the pseudo code of clusters construction phase:

For ( $n$ e $N, n=<N, i++)$ i

1. Listen the DiscoveryMessage from the BST with an DiscoveryMessageID fixed at 0

2. IF (DiscoveryMessageID=0) become a gateway node

3. Increment the ID before sending it to a neighbor node

4. IF (DiscoveryMessageID=3) become a $\mathrm{CH}$ and from here to the DiscoveryMessageID should be decremented in each reception until it becomes $=0$

The $C H$ node sends also in the a ClusterID $=1$ to be incremented only by the next $\mathrm{CH}$

5. While (the DiscoveryMessageID=0) become a gateway node

Repeat the process (line 3 to 5) Until $n=N$

if (single node) join the cluster with the last known CuusterID \}

In the end of this phase, three lists are formed:

- List_members: present in each $\mathrm{CH}$, contains the set of member nodes of a cluster.

- List_agregators: present in each $\mathrm{CH}$, gives an overall view of neighboring clusters.

- List_CH: present in the BST, list of CHs in the network. 
Once the clusters are formed, $\mathrm{CHs}$ receive data from simple nodes and creates TDMA tables based on the number of nodes that form the clusters. When all the data are received, they are compressed by the $\mathrm{CHs}$ and transmitted to the base station.

\subsubsection{TDMA Schema and Route Management}

Once the clusters are formed, the Base station uses a Msg_BTS to assign a MAC transmission code to each cluster in order to limit inter-cluster collisions, in their turn $\mathrm{CHs}$ assign a MAC transmission code to each member node to limit intra-cluster collisions. In addition, in each cluster a list of all the gateway nodes to the neighboring clusters is built and will be used in intercluster routing.

A route is defined by the triplet (SourceID, DestinationID, cost). Intra-cluster communications are operated simultaneously in all clusters of the network. For inter-cluster communication process, each $\mathrm{CH}$ broadcasts a message which contains its ID, residual energy, the number of his cluster members, and distance to the base station.

\subsubsection{Reelection of $\mathrm{CH}$ and the Metric used}

In each new round the election of the new $\mathrm{CH}$ doesn't pass through the BST but rather through the old $\mathrm{CH}$ according to three elements:

- Energy level of the new $\mathrm{CH}$.

- Density of the nodes at two hops.

- Distance from the old $\mathrm{CH}$.

Each node calculates its probability to be a $\mathrm{CH}$ using the fallowing metric:

$$
\mathrm{P}(\mathrm{CH})=\left[\alpha^{*}(\text { Res-energy })+\beta *(2-\text { Density })+\gamma *(\text { Distance from last } \mathrm{CH})\right]
$$

Where the 2-Density refers to the number of neighbouring nodes at two two-hop and the distance between two nodes $(i)$ and $(j)$ is denoted by:

$$
\operatorname{Distance}_{(i, j)}(t)=\sqrt{\left(X_{i}(t)-X_{i}(t)\right)^{2}+\left(Y_{i}(t)-Y_{j}(t)\right)^{2}}
$$

The values of the parameters $(\alpha, \beta, \gamma)$ depend on the application with : $\alpha+\beta+\gamma=1$

This approach avoids voting consumption, reduces the energy of clustering and assures $\mathrm{CH}$ rotation which helps to prolong network lifetime.

\subsubsection{Data Aggregation Model}

Our aggregation method is based on the Data centric approach used to solve the data implosion problem that characterizes the sensor networks enabling processing of redundant data at the time of transmission on the network.

In each cluster, sensor nodes of a level $(\mathrm{N})$ aggregate the data coming from the lower level nodes $(\mathrm{N}-1)$. In addition, a data aggregation process is performed between $\mathrm{CHs}$ during the inter-clusters communications. 


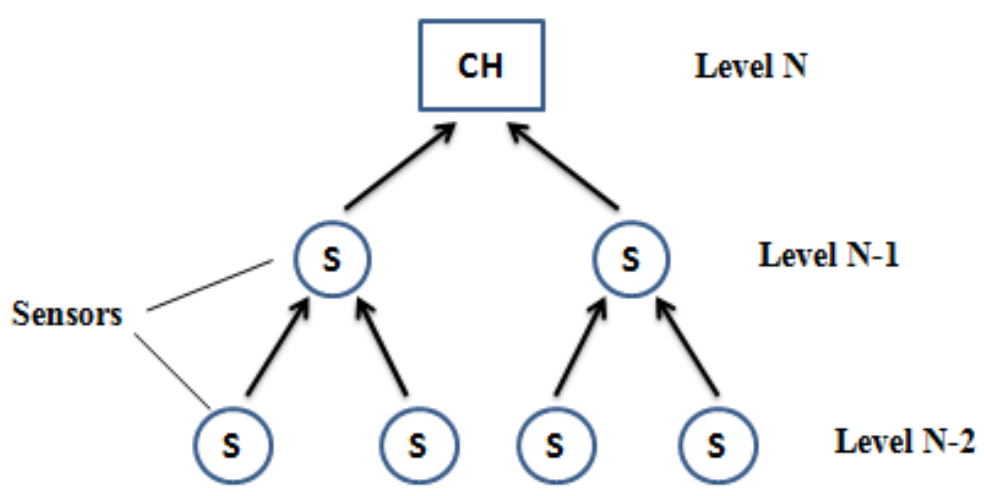

Figure 5. Data aggregation process in each cluster

The data aggregation on several levels represents an effective way to reduce the amount of information communicated and therby to decrease energy consumption and to improve network lifetime.

\subsubsection{Exchanged packets}

Our protocol uses three types of packets: HelloPacket to discover neighboring nodes and establish paths, ClusterConstructPacket used in the construction phase of the clusters and in the election of $\mathrm{CHs}$, DataPacket that contains the data to be transmitted in the network. Depending on the packet type, different informations are used in our protocol:

- Identifier of the source node, its $\mathrm{CH}$ and its coordinates (x, y)

- Identifier of the parent of the source node

- Calculated metric of the source node

- Number of hops between the source node and its $\mathrm{CH}$

- List of the source node neighbors and their coodinates

- List of the neighboring clusters addresses

- Identifier of the destination node and its ClusterID (DestinationClusterID)

- NexthopID : Nexthop node identifier

- Data: Transmitted data

- $\quad$ TTL Lifetime of the packet

\section{RESULTS AND DISCUSSIONS}

In this part, we compare our routing protocol with some efficient hierarchical routing protocols from the literature: LEACH, PEGASIS and HEED, and we present the results of our simulation based on evaluating metrics of delay, throughput, packet delivery ratio and energy level.

\subsection{Simulation parameters}

In our simulation, we distribute the sensors in the capture field with a sufficient deployment density to ensure connectivity on the network, the nodes are homogeneous and possess the same transmission radius and can send their data periodically. Our simulations have been run in MATLAB (Matrix laboratory), Table 1 lists the simulation parameters. 
Table 1: Simulation Parameters

\begin{tabular}{|c|c|}
\hline Parameters & Values \\
\hline Simulation area & $100 \mathrm{~m} \mathrm{X} \mathrm{100} \mathrm{m}$ \\
\hline Initial energy E0 & $0,5 \mathrm{Joules}$ \\
\hline $\begin{array}{c}\text { Energy consumed in the } \\
\text { electronics circuit, Eelec }\end{array}$ & $50 \mathrm{~nJ} / \mathrm{bit}$ \\
\hline $\begin{array}{c}\text { Energy consumed by the } \\
\text { amplifier Efs }\end{array}$ & $10 \mathrm{pJ} / \mathrm{bit} / \mathrm{m}^{2}$ \\
\hline Base station location & $(\mathrm{X}=0 \mathrm{~m} ; \mathrm{Y}=0 \mathrm{~m})$ \\
\hline Number of nodes & 100 \\
\hline Sensing rage & $5 \mathrm{~m}$ \\
\hline Transmission range & $2000 \mathrm{bits}$ \\
\hline Message size & $5000 \mathrm{bits} / \mathrm{sec}$ \\
\hline Bandwidth & $250 \mathrm{bits}$ \\
\hline Control packet & $100 \mathrm{bits} / \mathrm{sec}$ \\
\hline Data transmission speed &
\end{tabular}

\subsection{Results Analyzis}

In this part, we present our simulation results and we compare the performance of our protocol with the studied clustering protocols.

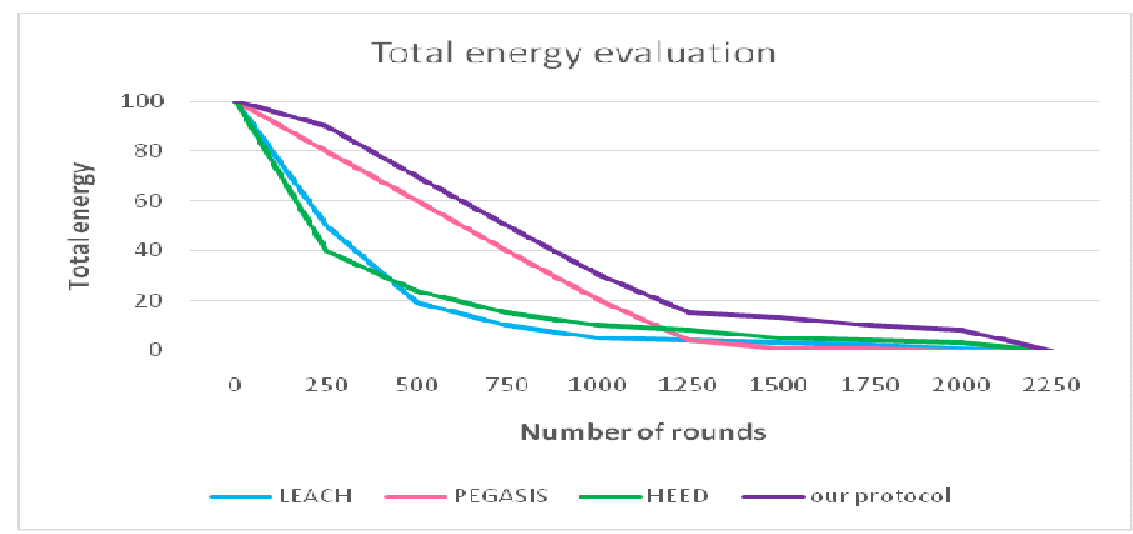

Figure 6. Total energy evaluation in our protocol Vs LEACH, PEGASIS and HEED

The total energy in each round is presented by the sum of residual energy of all the network nodes, the rotation of the $\mathrm{CH}$ role on all the nodes of the cluster allows to balance the energy consumption of the cluster, but in the meanwhile it generates an overconsumption of energy since each rotation of $\mathrm{CH}$ requires a diffusion phase to inform the nodes about the new $\mathrm{CH}$.

PEGASIS uses only one transmission to the BS per round and reduces the control overhead which improves its performance. In LEACH, the ability to communicate with the sink through any node on the network requires considerable energy consumption from distant nodes, so the aggregation of the data is centred at the level of the CHs, which makes them the weak links of the network. PEGASIS considers that all the nodes of the network can reach the sink, which requires an adjustable transmission with an important energy overhead.

Figure 6. shows that the energy decrease in our protocol is much slower than the other protocols (LEACH, PEGASIS and HEED) because it selects $\mathrm{CH}$ nodes according to the residual energy and 
to the minimal energy from the previous $\mathrm{CH}$ rather than random probability and that extends network lifetime .

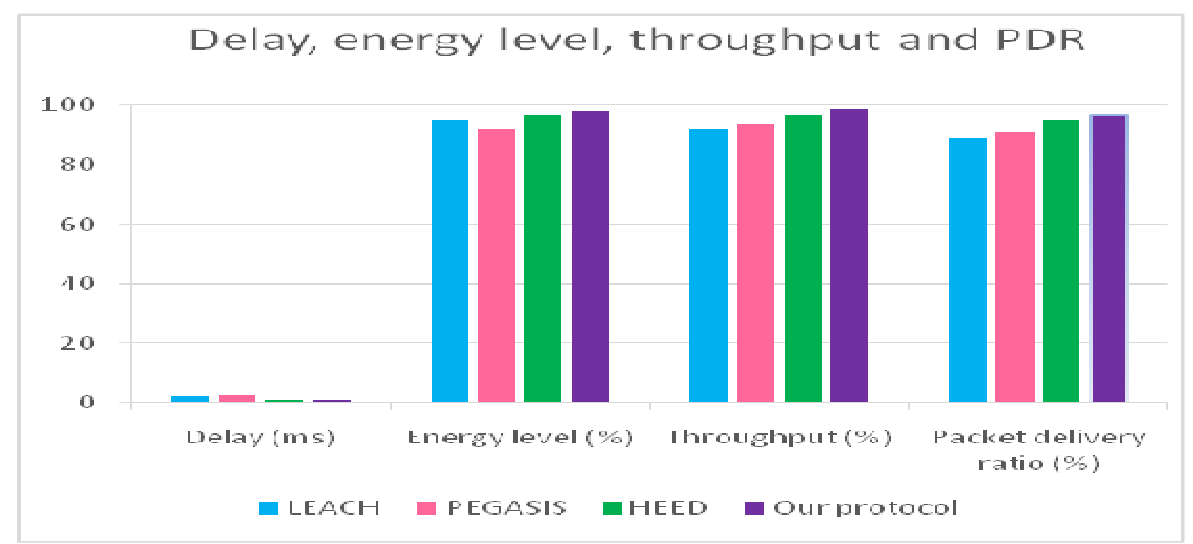

Figure 7. Performance analysis of our protocol Vs LEACH, PEGASIS and HEED

The packet delivery ratio is the number of packets delivered to the destination to the number of packets supposed to be received, the greater value of packet delivery ratio means the better performance of the protocol this ratio is suitable in all the tested protocols.

The throughput is the ratio of the number of packets sent to the total number of packets, the greater value of throughput means the better performance of the protocol.

In LEACH, energy consumption is shared across all nodes and the use of TDMA / CDMA techniques allows for a hierarchy and multi-level clustering, which saves more energy.

PEGASIS protocol try to eliminate of the overhead caused by dynamic cluster formation in LEACH but the length of the chain formed in PEGASIS protocol can considerably increase the data delivery time and the only node allowed to communicate with the BS can become a bottleneck within the network.

As it can be seen from (Figure 7.) our protocol has a minimal delay and an efficient throughput when compared with the other protocols. Since our protocol is executed in cooperation between all the nodes of the network, each node decides independently of its role which guarantees better distributed energy consumption. Moreover, the use of multi-hops routing guarantees a compromise between the size of the clusters and the number of clusters in the network which ensures energy balancing.

\section{CONCLUSIONS}

In this work, we investigated the study of hierarchical routing strategies in WSN, which allowed us to highlight their characteristics and limits. We compared some of the existing hierarchical energy efficient routing protocols with our routing protocol and we discussed the simulations results in term of delay, throughput, packet delivery ratio and energy consumption then we deduct that our routing scheme is more optimal and energy efficient. As a future work, we are working on the development of our protocol to improve its efficiency with other parameters then we will publish this work in a more detailed version of this paper. 


\section{REFERENCES}

[1] W. R. Heinzelman, et al., (2010) "Energy-efficient communication protocol for wireless microsensor networks," 33rd Annual Hawaii International Conference on System Sciences.

[2] S. Lindsey, C. Raghavendra, (2002) "PEGASIS: Power-Efficient Gathering in Sensor Information Systems", IEEE Aerospace Conference Proceedings, Vol. 3, 9-16 pp. 1125-1130.

[3] Gang Lu et al, (2008) "An Adaptive Energy-Efficient and Low-Latency MAC for Data Gathering in Sensor Networks", 2004. 9. Injong Rhee et al, "Z-MAC: a Hybrid MAC for Wireless Sensor Networks", IEEE/ACM Transactions on Networking (TON), Vol 16, Issue 3.

[4] R. C. Shah and J. Rabaey, "Energy Aware Routing for Low Energy Ad Hoc Sensor Networks", IEEE Wireless Communications and Networking Conference (WCNC), March 17-21, 2002, Orlando, FL.

[5] Ossama Younis and Sonia Fahmy "Distributed Clustering inAd-hoc Sensor Networks: A Hybrid, Energy-Efficient Approach for Ad-hoc Sensor Networks", Department of Computer Sciences, Purdue University.

[6] Falko Dressler and Ozgur B. Akan, '2010) "A survey on bio-inspired networking", The International Journal of Computer and Telecommunications Networking, vol. 54, issue 6, pp. 881-900.

[7] S. Banerjee and S. Khuller, A clustering scheme for hierarchical control in multi-hop wireless networks, in Proceedings of 20th Joint Conference of the IEEE Computer and Communications Societies.

[8] Dahlila P. Dahnil, Yaswant P. Singh and Chin Kuan Ho, "Energy-Efficient Cluster Formation in Heterogeneous Wireless Sensor Networks: A Comparative Study”, Feb. 13 16, ICACT 2011.

[9] Vishal Kumar Arora, Vishal Sharma, Monika Sachdeva (2016) “ A survey on LEACH and other's routing protocols in wireless sensor network", Optik - International Journal for Light and Electron Optics, Volume 127, Issue 16.

[10] Abbas Nayebi, Hamid Sarbazi-Azad (2011) " Performance modeling of the LEACH protocol for mobile wireless sensor networks", Journal of Parallel and Distributed Computing, Volume 71, Issue 6, Pages 812-821.

[11] Sudhanshu Tyagi, Neeraj Kumar (2013) "A systematic review on clustering and routing techniques based upon LEACH protocol for wireless sensor networks", Journal of Network and Computer Applications, Volume 36, Issue 2, Pages 623-645

[12] Vrinda Gupta, Rajoo Pandey (2016) “ An improved energy aware distributed unequal clustering protocol for heterogeneous wireless sensor networks", Engineering Science and Technology, an International Journal, Volume 19, Issue 2, Pages 1050-1058.

[13] Shijun He, Yanyan Dai, Ruyan Zhou, Shiting Zhao (2012) " A Clustering Routing Protocol for Energy Balance of WSN based on Genetic Clustering Algorithm”, IERI Procedia, Volume 2, Pages 788-793.

[14] M. Mehdi Afsar, Mohammad-H. Tayarani-N " Clustering in sensor networks: A literature survey”, Journal of Network and Computer Applications, Volume 46, November 2014, Pages 198-226.

[15] Md Azharuddin, Pratyay Kuila, Prasanta K. Jana (2015) "Energy efficient fault tolerant clustering and routing algorithms for wireless sensor networks “, Computers \& Electrical Engineering, Volume 41, Pages 177-190. 
[16] Ramnik Singh, Anil Kumar Verma (2017) "Energy efficient cross layer based adaptive threshold routing protocol for WSN", International Journal of Electronics and Communications, Volume 72, Pages 166-173.

[17] Shilpa Mahajan, Jyoteesh Malhotra, Sandeep Sharma (2014) “ An energy balanced QoS based cluster head selection strategy for WSN”, Egyptian Informatics Journal, Volume 15, Issue 3, Pages 189-199.

[18] Wang Ke, Ou Yangrui, Ji Hong, Zhang Heli, Li Xi (2016) "Energy aware hierarchical cluster-based routing protocol for WSNs", The Journal of China Universities of Posts and Telecommunications, Volume 23, Issue 4.

[19] Melike Yigit, V. Cagri Gungor, Etimad Fadel, Laila Nassef, Nadine Akkari, Ian F. Akyildiz. (2016) "Channel-aware routing and priority-aware multi-channel scheduling for WSN-based smart grid applications" Journal of Network and Computer Applications, Volume 71, Pages 50-58.

[20] Hang Shen, Guangwei Bai. (2016) "Routing in wireless multimedia sensor networks: A survey and challenges ahead”, Journal of Network and Computer Applications, Volume 71, Pages 30-49.

[21] Jalel Ben-Othman, Bashir Yahya. (2010) "Energy efficient and QoS based routing protocol for wireless sensor networks", Journal of Parallel and Distributed Computing, Volume 70, Issue 8, Pages 849-857.

\section{AUTHORS}

\section{Alaa Aadri}

PhD student and member of the TIAD Laboratory in the Faculty of Sciences and Techniques, Sultan Moulay Slimane University. Obtained a master degree in business intelligence in 2013, completed by a master degree in Information Systems Management, Institute of Business Administration IAE-Lyon - France in 2015. His current interests include Mobile Computing, Networking and intelligent Systems developing.

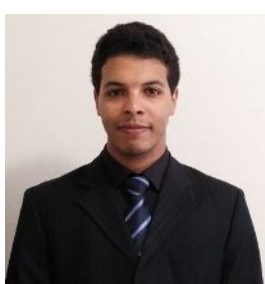

\section{Najlae Idrissi}

Member the TIAD Laboratory and professor in the Faculty of Sciences and Techniques, Sultan Moulay Slimane University in Beni mellal - Morocco. His current interests include Mobile computing and networking, signal, image and video processing and datamining.

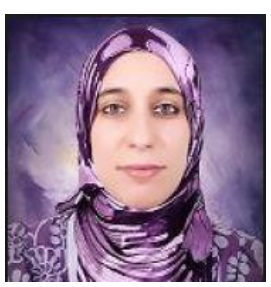

